\title{
Periodic orbits and equilibria in glass models for gene regulatory networks
}

\section{Report}

\section{Author(s):}

Zinovik, Igor; Chebiryak, Yury; Kröning, Daniel

Publication date:

2009

Permanent link:

https://doi.org/10.3929/ethz-a-005799174

Rights / license:

In Copyright - Non-Commercial Use Permitted 


\title{
Periodic Orbits and Equilibria in Glass Models for Gene Regulatory Networks
}

\author{
Igor Zinovik, Yury Chebiryak, Daniel Kroening
}

\begin{abstract}
Glass models are frequently used as models for gene regulatory networks. This paper proposes algorithmic methods for the synthesis of Glass networks with specific dynamics, including periodic orbits and equilibrium states. In contrast to existing work, bi-periodic networks and networks possessing both stable equilibria and periodic trajectories are considered. The robustness of the attractor is also addressed, which gives rise to hypercube paths with non-dominated nodes and double coils. These paths correspond to novel combinatorial problems, for which initial experimental results are presented. Finally, a classification of Glass networks with respect to their corresponding gene interaction graphs for the case of graphs with three edges is presented.
\end{abstract}

Index Terms-state transition diagram, interaction graph, wiring diagram, pathway, induced cycle, hypercube, dominating codes

\section{INTRODUCTION}

M ODELS of gene regulatory networks are often formulated as hybrid systems in which the switch-like behavior of genes is approximated by discontinuous step functions, while the other state variables still change continuously in time. A special class of piecewise-linear differential equations (PLDE) was proposed by Glass and Kaufman as a model approximation for the network dynamics in the context of gene regulation [3], [4]. These equations are applied to the analysis of gene regulatory networks [5], [6], [7], [8], and neural networks [9], [10].

A distinctive feature of biological phenomena is that the interactions are characterized by very localized coupling of the state variables, unlike complex couplings found in control and electronic circuit problems. In the resulting model, interactions between genes manifest only in the piecewise constant terms of the PLDE system [11]. The switch-like behavior of genes is modeled with the help of thresholds, which induce a partitioning of the PLDE phase space into a set of $n$-dimensional boxes where $n$ denotes the number of genes. In each box, the protein concentrations are described by ODEs with a constant production term $\mu_{i}$ and a rate parameter $\gamma_{i}$ :

$$
\dot{x}_{i}=\mu_{i}-\gamma_{i} x_{i} \quad \text { for } 1 \leq i \leq n
$$

This paper is an extension of two conference papers [1], [2]. A part of this work was presented at the $7^{\text {th }}$ Australia-New Zealand Mathematics Convention, Christchurch, New Zealand, December 11, 2008. The work was supported in part by ETH Research Grant TH-19 06-3 and by the ARTEMIS project CESAR.

I. Zinovik is with LTNT Laboratory of Thermodynamics in Emerging Technologies, ETH Zurich, 8092 Zurich, Switzerland.

Y. Chebiryak is with the Computer Systems Institute, ETH Zurich, 8092 Zurich, Switzerland.

D. Kroening is with the Computing Laboratory, Oxford University, UK.

where $x_{i}$ denotes the concentration of the product of gene $i$.

If the model of the gene activity is restricted to on/off expressions and the decay rates are identical for all reactions, the PLDE system is reduced to a Glass model [12]. Using appropriate scaling of the variables, the Glass PLDE can be transformed into the system

$$
\dot{y}_{i}=F_{i}\left(\tilde{y}_{1}, \ldots, \tilde{y}_{n}\right)-y_{i} \quad \text { for } 1 \leq i \leq n,
$$

where $\tilde{y}_{i}=0$ if $y_{i}<0$, and $\tilde{y}_{i}=1$ if $y_{i}>0$ [12]. These equations describe a network with all thresholds equal 0 and unit decay rate. The trajectories of the PLDE are straight lines in every orthant $\mathcal{O}_{k}, k \in\left\{1,2,3, \ldots, 2^{n}\right\}$, of the phase space. The phase flow in each orthant $\mathcal{O}_{k}$ is defined by its focal point $\boldsymbol{f}^{(k)}=\left(f_{1}^{(k)}, f_{2}^{(k)}, \ldots, f_{n}^{(k)}\right) \in \mathbb{R}^{n}$ where $f_{i}^{(k)}=\left.F_{i}\left(\tilde{y}_{1}, \tilde{y}_{2}, \ldots, \tilde{y}_{n}\right)\right|_{\mathcal{O}_{k}}$. Thus, the Glass network can be fully specified by a choice of focal points $\boldsymbol{f}^{(1)}, \ldots, \boldsymbol{f}^{\left(2^{n}\right)}$.

The phase flow in Glass networks is studied using a state transition diagram, which is represented by an $n$-dimensional cube with directed edges. Each orthant of the phase space is associated with a vertex of the $n$-cube, and each common boundary of the orthants corresponds to an edge of the cube. This edge is directed according to the direction of the phase flow across the boundary [13]. Figure 1 illustrates a phase flow with two trajectories in a two-dimensional Glass network. The state transition diagram for a 3-dimensional Glass network is shown in Fig. 2. The vertices of the $n$-cube are labeled by vectors of $n$ binary variables $\left(\tilde{y}_{1}, \tilde{y}_{2}, \ldots, \tilde{y}_{n}\right)$, which define a valuation of the network interaction functions $F_{i}$. Periodic trajectories of a network correspond to closed cycles in the transition graph (as an example, consider the thick path in Fig. 2). This connection enables reasoning about the phase flow in the Glass networks by examining the set of paths on the hypercube, a problem of independent importance in coding theory. In particular, cycles in the transition diagrams correspond to necessary conditions for the existence of periodic orbits in Glass networks.

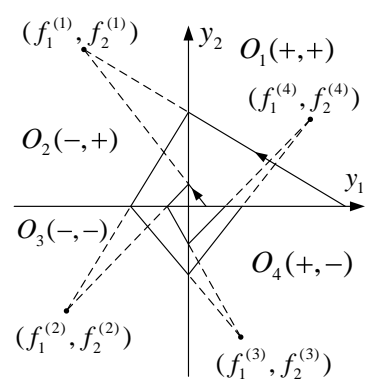

Fig. 1. A 2D phase flow

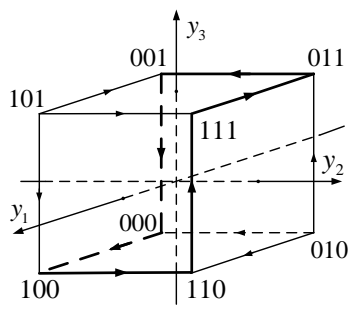

Fig. 2. A 3D transition diagram 
The global phase flow in Glass networks can be quite complex. Oscillations towards equilibrium states, cycles and limit cycles may occur when linear parts of the trajectories are connected continuously over sequences of orthants [13], [14], [12], [15]. Numerical simulations [13], [16] indicate that for dimensions greater than 4, Glass networks may exhibit aperiodic and chaotic behavior. Studies of the periodic solutions for Glass models show that there are networks that possess a special type of stable limit cycles: the flow between the orthants along these cycles is unambiguous, i.e., for each orthant along the cycle, all trajectories must go to the same successor. In other words, the basin of attraction of the periodic trajectory is composed of all orthants spanned by the trajectory. Networks with such stable cycles are called networks with cyclic attractors [13], which is formalized as follows.

Definition 1 (Cyclic Attractor): A cycle in the state diagram is called a cyclic attractor if a) it is a chord-free simple cycle in the $n$-cube ${ }^{1}$, and b) all edges adjacent to the cycle are directed towards the cycle nodes.

As an example, the cycle shown in Fig. 2 is the cyclic attractor.

Models for gene regulatory networks with equilibrium states and stable limit cycles are of special interest in Systems Biology as the models simulate cell differentiation processes and explain the variability of cell types [17], [18]. Simulations of cell division cycles require models with periodic orbits while models for the dynamics of cellular differentiation have to possess multiple stable (equilibrium) points.

Various approaches to the search for the equilibrium states in a variety of gene regulatory networks have been presented in the literature. In PLDE systems, the search for the equilibrium states and for periodic trajectories in the models of networks is known to be computationally demanding due to the combinatorial explosion of the size of the search space [15]. In case of PLDE with equilibrium states, a scalable search algorithm has been proposed recently by de Jong and Page [19]. Their method reduces the search to a first-order-logic satisfiability problem and relies on the heuristics implemented in the stateof-the-art solvers for SAT modulo theories (SMT).

The existing efforts to deduce fully defined dynamic models of gene regulatory systems are limited by the lack of experimental data on the kinetic constants of the biochemical reactions. Nevertheless, the models can be used to infer qualitative information about the interaction of the genes. Interaction is one of the most important properties of gene regulatory systems and is usually presented in the form of a wiring scheme. A wiring scheme is a directed graph that specifies inhibiting and activating interactions between the genes. In systems biology, the connection between qualitative dynamics of ODE-based models and their wiring schemes has been a research focus since the pioneering work of Thomas and Kaufman [17]. The results have been summarized as conjectures that link the equilibria and the periodic behavior of autonomous ODE to inhibiting and activating patterns of wiring schemes [20].

\footnotetext{
${ }^{1}$ Every edge in the graph that joins two vertices of the cycle is an edge of this cycle.
}

\section{Contribution}

The core contribution of this paper is the application of codes on binary hypercubes to reason about the behavior of Glass PLDE. We obtain the hypercube codes by means of modern satisfiability solvers [21], [22]. The utility of this reduction is demonstrated by three results:

1) In most of the existing literature, the discussion of the dynamics of Glass PLDE is restricted to either the case of a single stable periodic orbit or multiple equilibria. As our first contribution, we address instances of Glass networks of two types: a) with stable bi-periodicity and b) with co-existent equilibrium and stable periodic trajectory. Our reduction to hypercube paths enabled a full classification of the six-dimensional networks with a cyclic attractor with respect to the number of equilibrium states co-existent with the attractor. We have also synthesized instances of all networks with double attractors up to dimension seven and present upper bounds on the total length of the attractors.

2) In contrast to the models with continuous ODE, the wiring schemes of PLDE and their connection to the system dynamics have not been studied systematically. We have extended our SAT-based approach to the construction of Glass networks that correspond to a given wiring scheme. We also present a classification of the transition diagrams of the networks with respect to the wiring schemes of the models. To the best of our knowledge, it is the first classification of this kind for the Glass PLDE.

3) Sufficient conditions for periodic orbits in Glass networks require the computation of eigenvalues and eigenvectors of the matrix that specifies the Poincare return map of the phase flow [12]. Based on those criteria, we have developed an algebraic method for the synthesis of Glass networks with periodic orbits along given cycles in the transition diagram. As an exemplar, we construct all three-dimensional Glass networks with different periodic orbits without cyclic attractors. Finally, we suggest two conjectures about the transition diagrams, which can be seen as colloraries of conjectures for ODE systems [20].

\section{Outline}

In Sec. II, we discuss Glass networks that are appropriate to model cell division. The corresponding hypercube paths give rise to two novel novel combinatorial problems. In Sec. III, discuss wiring diagrams for Glass PLDE, and examine conjectures for models with continuous ODE in this context. In Sec. IV, we suggest an algebraic method for the construction of Glass PLDE with periodic orbits that avoids the complications connected with numerical methods.

\section{GLASS NETWORKS WITH COMPLEX ATTRACTING SETS}

\section{A. Glass networks for cell division}

Gene networks that regulate cell division may undergo a cyclic transformation of the gene expressions. Mathematical models capture this cyclic nature of the cell development using 
periodic dynamics. A normal development of the cell division should eventually stop with a tissue consisting of a finite number of cells, thus suggesting that the model has to possess the ability to describe both periodic and equilibrium states. For example, the vulval development of $C$. elegans exhibits a series of cell divisions with 22 nuclei finally formed. The cell division process reveals dynamics of a complex reactive system that includes at least four different molecular signaling pathways [23].

In the corresponding Glass model, the state of every signaling pathway is described by valuation of a Boolean variable, and the transition diagram is a four-dimensional cube. The diagram has to contain both a cyclic attractor (representing the switching of gene expressions during the cycles of cell division) and a node that specifies the final state of the system. All edges of this final node have to be directed inwards to ensure that the phase flow has a stable equilibrium in the corresponding orthant of the phase space of the Glass PLDE. This implies that the Hamming distance between the equilibrium node and the nodes of the cyclic attractors has to be greater than one. Otherwise, the orientation of at least one of the edges of the equilibrium node will be inconsistent with the definition of a cyclic attractor (Def. 1). We say that such a node is non-dominated or shunned by the cycle [2]. A formal definition follows.

Definition 2: The cycle $I_{0} \ldots I_{L-1}$ shuns node $W$ of the $n$-cube if $W$ is not adjacent to any node of the cycle:

$$
\forall j \in\{0, \ldots, L-1\} . \quad d_{H}^{n}\left(I_{j}, W\right)>1,
$$

where $d_{H}^{n}(a, b)$ denotes the Hamming distance between nodes $a$ and $b$ of the $n$-cube.

An example of a four-dimensional transition diagram with a cyclic attractor and an equilibrium node is given in Fig. 3. The orthants for the focal points of the PLDE are defined by the orientation of the diagram edges. A way to ensure the existence of a periodic orbit is to require focal coordinates of 1 or $-1[24] .^{2}$

The presence of nodes non-dominated by the induced cycle also indicates that the phase flow along the attractor is robust to arbitrary perturbations of the coefficients that define the equations in the orthant corresponding to the node. This robustness implies that the periodic behavior of the gene network will not be affected by the (likely unknown) protein production rate in any state corresponding to the non-dominated node.

We have computed all non-dominated nodes for every equivalence class of cyclic attractors up to dimension 6 . The details of the propositional encoding and the setup of the propositional SAT solver are beyond the scope of this article. We refer the interested reader to [2]. The results for the known instances of attractors in dimension 7 indicate that some cyclic attractors with the longest period may shun up to three nodes. Table I summarizes these results for the longest attractors and the attractors with a maximum number of non-dominated nodes. The partitioning of the equivalence classes of cyclic attractors with respect to the number of non-dominated nodes they admit is presented in Table II and Fig. 4.

\footnotetext{
${ }^{2}$ The Glass networks with such focal points are called Boolean Glass networks.
}

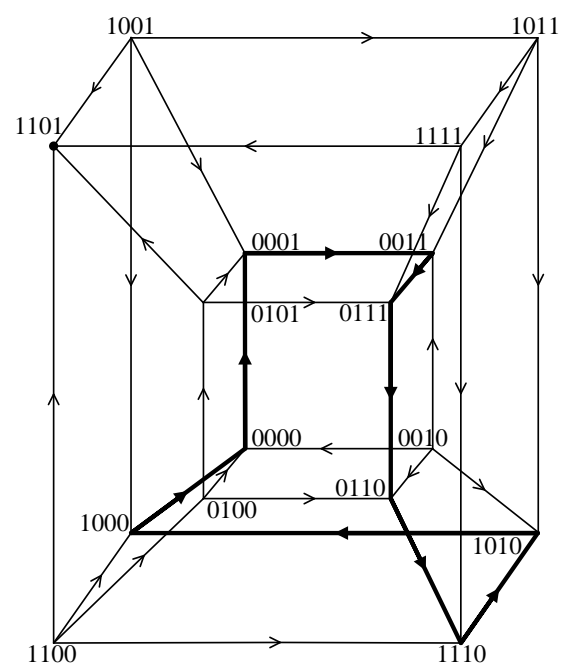

Fig. 3. An induced cycle in the 4-cube. The cycle shuns node 1101.

TABLE I

THE NUMBER OF NON-DOMINATED NODES FOR THE LONGEST CYCLIC ATTRACTORS

\begin{tabular}{c|c|c}
\hline \hline dimension $n$ & max length $L$ & \#non-dominated nodes \\
\hline \hline 3 & 6 & 0 \\
4 & 8 & $\{0,1\}$ \\
5 & 14 & 0 \\
6 & 26 & 0 \\
7 & 48 & $\{0,1,2,3, ?\}$ \\
\hline \hline
\end{tabular}

In Figure 4, we also report the classification for two new equivalence classes of six-dimensional transition diagrams. We have previously reported a classification of the induced cycles of length 16, which exceeds the maximal length of the induced cycles in cubes of dimension five [25]. However, it is possible to construct the induced cycles of length less than the maximal length in previous dimensions, which nevertheless cannot be found in cubes of lower dimensions. The minimum length of such a cycle in a cube of dimension $n$ cannot be less than $2 n$, because the cycle has to traverse all dimensions of the cube. In dimensions greater than 6 , an example of such a cycle can be given by the following sequence of node coordinates switching along the cycle: $(1, \ldots, n, 1 \ldots, n)^{3}$

\section{B. Multiperiodic Glass networks}

An important feature of models of gene networks with chaotic dynamics is multiperiodicity [20]. The transition diagrams of such multiperiodic systems have to contain multiple cycles. If a gene network exhibits dynamics with multiple stable periodic orbits, a Glass PLDE with multiple cyclic attractors may be a suitable model. The transition diagrams of these Glass networks have to possess multiple induced cycles.

\footnotetext{
${ }^{3}$ It is easy to see that a cycle with such coordinate sequence of length $L=2 n$ is indeed an induced cycle, as the sequence satisfies the criterium given in [4]: 1) Each coordinate in the sequence must appear an even number of times. 2) For any sequence of length shorter than $L$, at least one coordinate must appear an odd number of times. 3) Every sequence of consecutive digits of length $I$, where $I$ is an odd integer $3 \leq I \leq L-3$, must contain at least 3 coordinates that appear an odd number of times.
} 
TABLE II

THE NUMBER OF EQUIVALENCE CLASSES OF CYCLIC ATTRACTORS

\begin{tabular}{|c|c|c|c|}
\hline dimension $n$ & length $L$ & \#non-dominated nodes & \#cycles \\
\hline \multirow[t]{7}{*}{5} & \multirow[t]{7}{*}{10} & 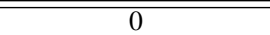 & 0 \\
\hline & & 1 & 0 \\
\hline & & 2 & 3 \\
\hline & & 3 & 3 \\
\hline & & 4 & 3 \\
\hline & & 5 & 0 \\
\hline & & 6 & 1 \\
\hline \multirow[t]{5}{*}{5} & \multirow[t]{5}{*}{12} & 0 & 2 \\
\hline & & 1 & 0 \\
\hline & & 2 & 2 \\
\hline & & 3 & 0 \\
\hline & & 4 & 1 \\
\hline 5 & 14 & 0 & 3 \\
\hline \multirow[t]{9}{*}{6} & \multirow[t]{9}{*}{12} & 16 & 16 \\
\hline & & 17 & 10 \\
\hline & & 18 & 15 \\
\hline & & 19 & 9 \\
\hline & & 20 & 5 \\
\hline & & 21 & 1 \\
\hline & & 22 & 0 \\
\hline & & 23 & 0 \\
\hline & & 24 & 1 \\
\hline \multirow[t]{9}{*}{6} & \multirow[t]{9}{*}{14} & 10 & 16 \\
\hline & & 11 & 13 \\
\hline & & 12 & 25 \\
\hline & & 13 & 26 \\
\hline & & 14 & 30 \\
\hline & & 15 & 12 \\
\hline & & 16 & 13 \\
\hline & & 17 & 1 \\
\hline & & 18 & 1 \\
\hline \multirow[t]{17}{*}{6} & \multirow[t]{17}{*}{16} & 0 & 1 \\
\hline & & 1 & 0 \\
\hline & & 2 & 1 \\
\hline & & 3 & 1 \\
\hline & & 4 & 13 \\
\hline & & 5 & 14 \\
\hline & & 6 & 44 \\
\hline & & 7 & 60 \\
\hline & & 8 & 108 \\
\hline & & 9 & 105 \\
\hline & & 10 & 111 \\
\hline & & 11 & 53 \\
\hline & & 12 & 34 \\
\hline & & 13 & 7 \\
\hline & & 14 & 8 \\
\hline & & 15 & 1 \\
\hline & & 16 & 2 \\
\hline
\end{tabular}

We have extended our propositional encoding [25] of single induced cycles in order to perform a search for multiple cycles. We have identified all hypercubes with two cyclic attractors up to dimension seven (Table III). The combined length of two attractors was found to be less than the maximum length of the single attractor in the cubes up to dimension six. In dimension seven, the combined length reaches the maximum length of the single cycle. An exhaustive search was feasible up to dimension six, which implies that the results in the table are indeed tight: there are no cubes with a combined length of the two cycles exceeding those that are shown in the table.

The results presented above for the complex attracting sets in the Glass networks give a rise to two combinatorial problems related to paths in hypercubes. The problems can be formulated as follows:
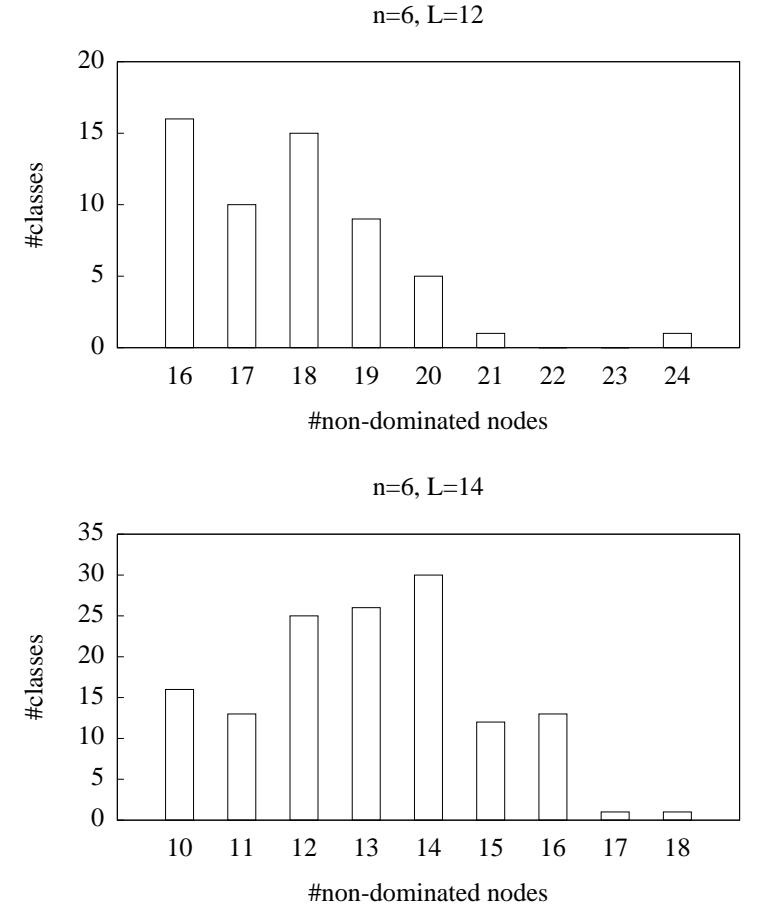

Fig. 4. The classification of cyclic attractors in 6-dimensional hypercubes with a given length $L$ with respect to the number of non-dominated nodes

1) What is maximum number of unspanned nodes in hypercubes with an induced cycle of given length $L$ ?

2) What is the combined length of multiple induced cycles in a cube of given dimension $n$ ?

The co-existence of a longest cyclic attractor and an unspanned node in the model suggests that during cell division, the gene network may traverse a maximum possible number of different states before switching to the final equilibrium. The combined length of multiple induced cycles is an upper bound on the number of states of the multiperiodic system.

\section{TRAnsition Diagrams AND Wiring Schemes}

\section{A. Wiring schemes}

An analysis of gene interactions is almost always part of mathematical simulations of gene regulatory networks. Wiring schemes or interaction graphs are the most common way to present information on the interaction between genes. Formally, a wiring scheme is an oriented and labeled graph whose vertices represent the variables of the system. The graph specifies inhibiting and activating interactions between the genes. We write $g_{1} \longrightarrow g_{2}$ if $g_{1}$ activates $g_{2}$, and $g_{1} \longrightarrow g_{2}$ if $g_{1}$ inhibits $g_{2}$.

The interaction between genes is often deduced by solving an inverse problem for a chosen model of the system. The Glass PLDE can serve as the underlying model if the experimental data series are sufficiently long [26]. Nevertheless, a systematic study of the interaction graphs related to PLDE is rarely presented in the literature. To the best of our knowledge, the only study addressing the link between the phase flow of PLDE and their wiring scheme is presented in [27]. Their 
TABLE III

THE CUBES WITH TWO CYCLIC ATTRACTORS

\begin{tabular}{c||c||c|c|c||c|c|c}
\hline \hline \multirow{2}{*}{ Dimension $n$} & max length $L$ & \multicolumn{4}{c||}{ First attractor } & \multicolumn{3}{c}{ Second attractor } \\
\cline { 3 - 8 } & & length & start node & coordinate sequence & length & start node & coordinate sequence \\
\hline \hline 4 & 8 & 4 & 1111 & 4242 & 4 & 0010 & 4242 \\
5 & 14 & 6 & 01010 & 153153 & 6 & 00100 & 513513 \\
6 & 26 & 20 & 001110 & 26536156254356251245 & 4 & 000010 & 2626 \\
7 & 48 & 24 & 0011000 & 124165716417347562574563 & 24 & 1111111 & 312517234523761571374562 \\
\hline \hline
\end{tabular}

result states that if the wiring scheme is a cycle with one negative interaction, it is always possible to construct a PLDE system with stable periodic orbit. In contrast, the wiring schemes of ODE with continuous coefficients are widely studied and well understood (see [20] for further references). The results can be formulated in the form of conjectures that link equilibriums and periodic behavior of autonomous ODE to inhibiting and activating patterns of the gene interaction.

\section{B. $O D E$ vs. PLDE}

The gene interaction for ODE with continuous coefficients is defined by the signs of the elements of Jacobian matrix $\|J\|$ of the system [20]. If $J_{i j}>(<) 0$, then gene $j$ activates (inhibits) gene $i$. The edges of the wiring scheme are labeled by the sign of the corresponding Jacobian element. The wiring scheme is said to have a positive (negative) circuit in a point $\mathbf{x}$ of the state space if a product of $J_{i j}$ with cyclical permutation of indexes $(i, j)$ is positive (negative).

The conjectures about the circuits of the wiring schemes have been formulated as follows:

1) The presence of a positive circuit (somewhere in the phase space) is a necessary condition for multistationarity.

2) The presence of a negative circuit of length at least two (somewhere in the phase space) is a necessary condition for stable periodicity.

We investigate whether these conjectures are valid in the case of PLDE systems. As a first step, we identify the differences between the modeling of gene interactions in PLDE systems and in ODE with continuous coefficients. In the PLDE, the Jacobian is not defined on the threshold hyperplanes, and thus by definition, $i \longrightarrow j$ implies that an increase of $x_{i}$ may lead to an increase of the kinetic rate of protein production linked to $\mu_{j}$ at least at some point $\mathbf{x}$ in the state space [27]. If the increase of the concentration leads to a decrease of the kinetic rate, this is depicted as $i \longrightarrow j$. For the Glass PLDE, the definition is consistent with the definition of the schemes for ODE with continuous coefficients, with two noteworthy differences:

1) If the Jacobian-based definition is applied to the Glass PLDE within the coordinate orthants, the wiring scheme will contain self-inhibiting edges at every node of the scheme whereas the definition of the PLDE wiring scheme does not require any self-regulating edges.

2) In contrast to Jacobian-based schemes, which characterize the interactions for a given local point, the PLDE wiring schemes present all local interactions on one graph.
To analyze the conjectures above in the context of PLDE, we need a definition of positive (negative) circuits in PLDE wiring schemes that is consistent with the definition for ODE with continuous coefficients and does not require the computation of derivatives. We propose the following definition: a circuit is called positive (negative) if the circuit contains an even (odd) number of inhibiting edges.

We apply our encodings of hypercube paths to check the conjectures for the Glass networks as follows: In Glass networks, multistationarity in the phase flow is equivalent to the presence of two or more equilibrium nodes in the oriented hypercubes. If the hypercubes contain a cyclic attractor, the Glass PLDE admits a stable periodic orbit. Therefore, a classification of the hypercubes with equilibria and attractors with respect to their wiring schemes could possibly provide a counterexample for the conjectures. In the following, we first suggest a number of applications and then discuss the results of a full classification for gene networks with three genes.

\section{Applications}

It is known that the search over oriented hypercubes is computationally demanding due to the rapid explosion of the search space. For example, in the four dimensional case, the number of the cubes reaches $2^{32}$ and search is usually performed using random sampling [15]. For our classification, we obtain the set of hypercubes for a given wiring scheme as a solution of a propositional satisfiability problem.

Our encoding is based on a set of independent Boolean variables that define the labels of the nodes and orientations of the edges. These variables are used to introduce a set of dependent Boolean variables that represent the signs of focal points and the interactions between the genes. The dependent variables are defined as functions of the variables for the labels and the orientations according to the definitions of the focal points and the gene interactions. The wiring scheme of interest is encoded as Boolean constraint such that satisfiable assignments identify the transition diagrams of the networks.

Example: As an illustrative example, we have identified the transition diagrams of the Glass networks with the wiring schemes that represent the interactions of the genes of $C$. elegans presented in [28]. Several alternative diagrams of the gene interactions are suggested to explain the formation of the cell patterns with two distinct fates. The interaction graphs that can be deduced from these diagrams are shown in Figure 5.

We performed a search over all $2^{12}$ oriented 3D cubes with the following constraints: 1) every edge of the wiring schemes has to appear at least once and 2) no edges except those shown are allowed. The search with our propositional 


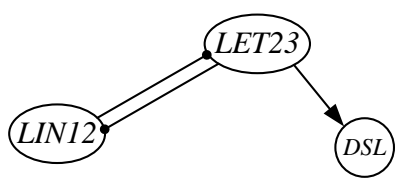

(a)

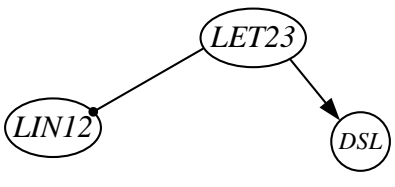

(b)
Fig. 5. Two candidate wiring schemes for the vulval development of C. elegans, derived from [28]

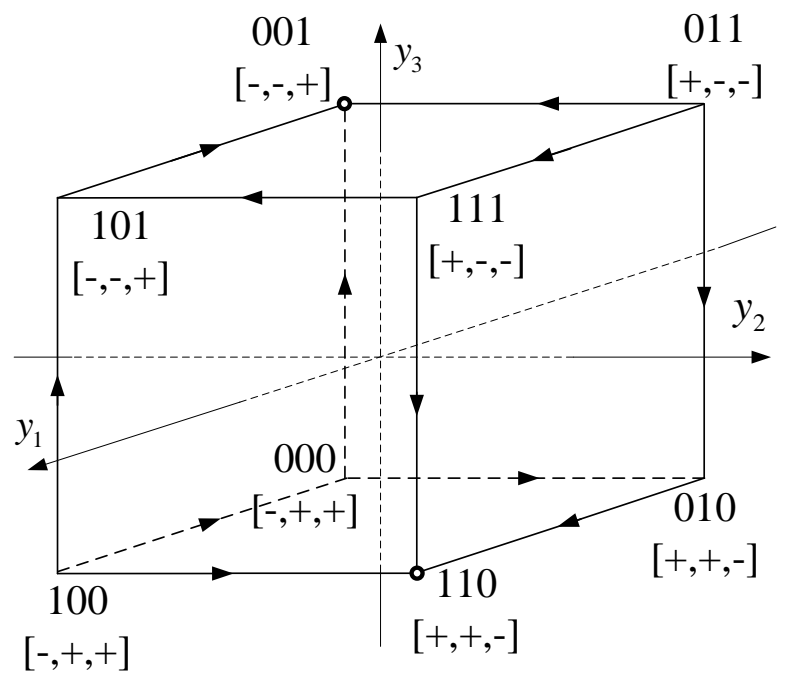

Fig. 6. A unique cube complying to the wiring scheme in Fig. 5 (a). Signs of focal points are denoted in brackets. Dimensions 1, 2 and 3 correspond to signaling pathways DSL, LET23, and LIN12 respectively.

satisfiability encoding of the problem required 7 seconds (see Table $\mathrm{V}$ in the Appendix for the full results). The search shows that for the scheme in Fig. 5 (a), there is only one transition diagram and that it has two equilibrium nodes (it is depicted in Figure 6). The second wiring scheme (Fig. 5 (b)) was found to admit two oriented cubes, each of which has a single equilibrium node. These results indicate that the cell dynamics of $C$. elegans with its two distinct stationary states cannot be modeled using the second scheme. This conclusion is consistent with the conjecture about multistationarity, i.e., the first scheme has the positive circuit $1 \longrightarrow 2 \longrightarrow 1$, which manifests the necessary condition of multistationarity whereas the second wiring scheme does not have the circuits.

Wiring diagrams with variable edges: An important property of gene networks is the ability of interaction between two genes to change from inhibition to activation and vice versa, depending on the expressions of the genes [29]. In PLDE systems, the wiring schemes of such networks will contain an edge that has different signs in different nodes of the transition diagram. Following the definition for ODE with continuous coefficients [20], we call such edges variable edges. The presence or lack of variable edges in a wiring scheme may serve as another classification criterion for transition diagrams.

As an example of such a classification, we have computed how many cyclic attractors induce wiring schemes with variable edges. The variable edges in the schemes were identified using the coordinate sequences of the equivalence classes of the attractors obtained in [25]. The results for the attractors

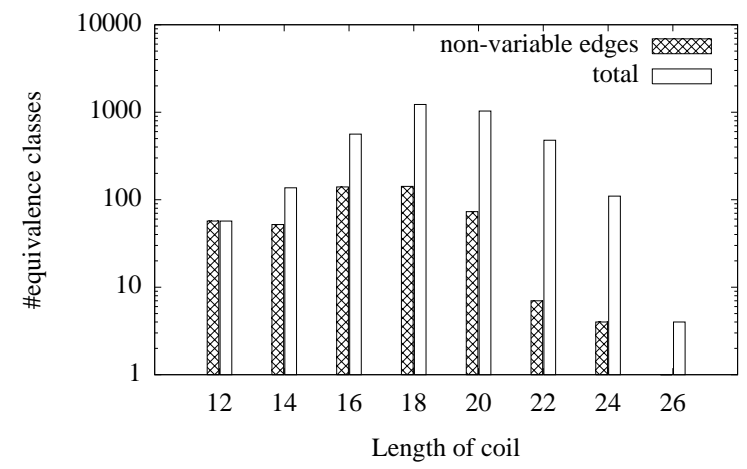

Fig. 7. Classification of coils with respect to variable edges of wiring scheme

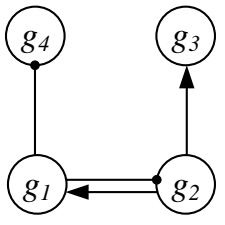

(a) Wiring diagram

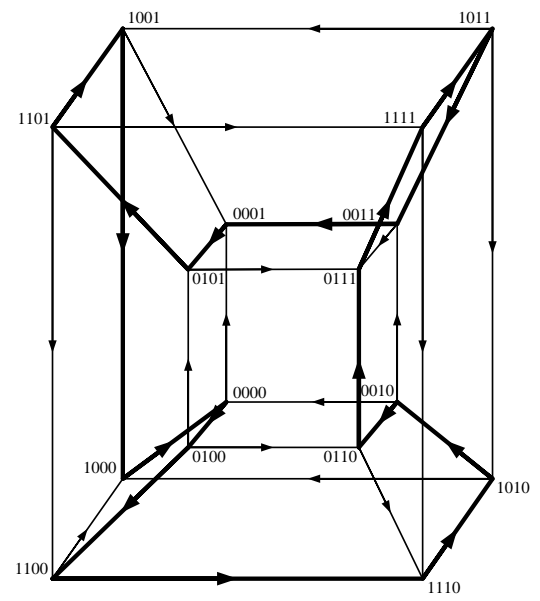

(b) Boolean Glass network
Fig. 8. A sample Boolean Glass network with Hamiltonian path and its wiring scheme

are summarized in Table IV and Figure 7. We found that the relative number of the equivalence classes with variable edges increases with an increasing length of the attractor, and that in dimensions five and six, the wiring schemes of all attractors of maximum period have variable edges. This result implies that in the Glass model, stable periodic orbits of maximum length cannot be described by the wiring schemes without variable edges.

Note that this classification of the attractors is based on the coordinate sequences along the nodes of the attractors. It is possible to construct different transition diagrams with attractors from the same equivalence class in such a way that one of the diagrams induces a wiring scheme without variable edges and other one will have at least one variable edge. Our satisfiability encoding provides a flexible tool for the synthesis of transition diagrams with prescribed properties for their wiring schemes. As an example, we constructed a four-dimensional oriented cube with an oriented Hamiltonian cycle where the wiring scheme does not contain any variable edges (Fig. 8).

In order to further evaluate the scalability of our SATbased search, we have constructed five-dimensional oriented hypercubes that conform to the wiring scheme in Fig. 9. This wiring scheme is adopted from [30] and outlines relationships 
TABLE IV

ATTRACTORS INDUCING WS WITH VARIABLE EDGES

\begin{tabular}{c|c|c|c|c|c}
\hline \hline Dimension & Length & \#WS without variable edges & \%WS without variable edges & \#coils (total) & \#WS with variable edges \\
\hline \hline 6 & 12 & 57 & 100 & 57 & 0 \\
& 14 & 52 & 38 & 137 & 85 \\
& 16 & 140 & 25 & 563 & 423 \\
& 18 & 142 & 12 & 1228 & 1086 \\
& 20 & 73 & 7 & 478 & 959 \\
& 22 & 7 & 1 & 110 & 471 \\
& 24 & 4 & 4 & 4 & 106 \\
& 26 & 0 & 0 & 10 & 0 \\
\hline 5 & 10 & 10 & 100 & 5 & 3 \\
& 12 & 2 & 40 & 3 & 3 \\
\hline 4 & 14 & 0 & 0 & 3 & 0 \\
\hline \hline
\end{tabular}

among the principal molecular components of the cell cycle engine during the cell division.

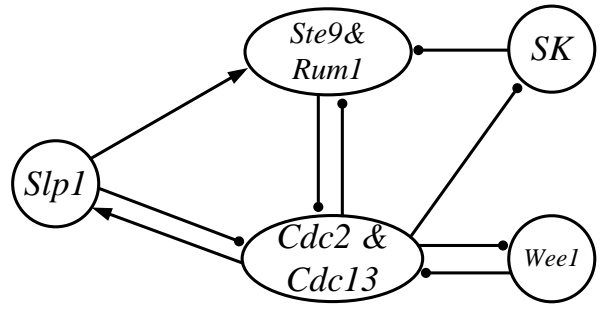

Fig. 9. The wiring diagram of eukariotic cell division [30].

Since the scheme models periodic changes of the concentrations of the cell proteins, we have implemented a search for the transition diagrams that possess at least one simple or complex cycle of a prescribed length. We encode the problem as a propositional SAT formula. The encoding leaves the choice of the orientation of the edges to the SAT solver. The orientations are only constrained by the given wiring scheme: their choice depends on the choices of incident edges and restrictions on focal points dictated by a wiring scheme. We restrict the cube to have a cycle of a prescribed length using the encoding of simple cycles by Papadimitriou (see example 8.1 in [31] for Hamiltonian cycles in arbitrary graphs). This simple cycle may be a part of a complex cycle.

In order to identify how many cycles are there in the resulting hypercube, we employ a backtracking algorithm proposed by Szwarcfiter and Lauer [32], which improves the asymptotically fastest algorithm algorithm by D. B. Johnson [33]. We identify strongly connected components using Tarjan's algorithm [34]. The results of the computations are summarized in Table VI (in the Appendix), listing all possible configurations (i.e. the number of complex cycles and equilibria, see also Figure 10). The results show that the algorithm is able to perform an exhaustive search up to cycle length 32 in less than 16 hours of CPU time.

Figure 10 and Table VI (in the Appendix) present results of the run of our algorithm on cubes complying to the scheme in Fig. 9 for all lengths of cycles from 4 to 32 .

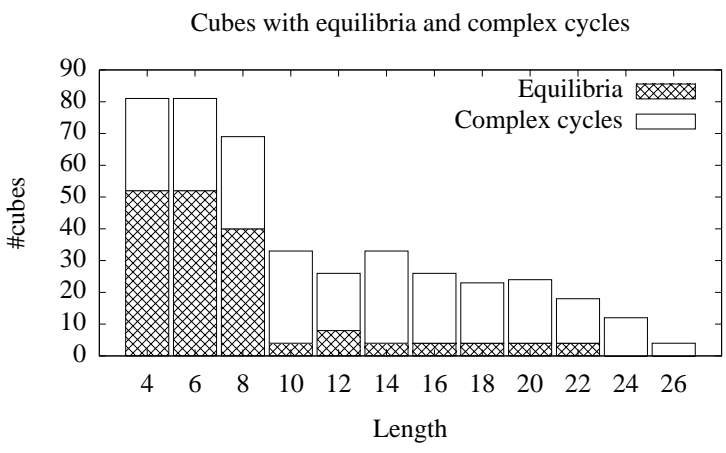

Fig. 10. Hypercubes with complex cycles and equilibria

\section{A full classification for networks with three genes}

In System Biology, a classification of the networks in lower dimensions is also desirable, owing to the possibility of elucidating the fundamental links between the system dynamics and the elements of complex gene interactions. In [20], a full classification of the possible dynamics of ODE with continuous coefficients with respect to the wiring schemes is shown for two-gene networks. To the best of our knowledge, there is no report of such a classification for the Glass PLDE model.

As a first step of a comprehensive classification of the Glass networks, we suggest to begin with the analysis of three interacting genes. We have applied our SAT-based search for the synthesis of the transition diagrams for all possible wiring schemes with three non-variable edges. An exhaustive conditional search was carried out assuming that in all wiring schemes, every edge has to be enforced at least at one node of the transition diagram and that there are no other edges in the scheme except those shown. The schemes and their diagrams are presented in the Appendix. We observe that the full set comprises of 28 wiring schemes that comply with 67 oriented cubes. A total of 15 wiring schemes correspond to a unique oriented cube, and not more than 4 cubes with the same scheme can be constructed in the set. Furthermore, a total of 24 transition diagrams have simple or complex cycle and 10 among them include cyclic attractors. Single equilibrium nodes were found in 39 cubes, whereas 14 diagrams possess two 
equilibrium nodes. In those cubes with cyclic attractors and double equilibrium nodes, the wiring schemes have negative or positive circuits, respectively. Since in both cases of the transitions in the diagrams express sufficient conditions for the corresponding dynamics of the Boolean Glass PLDE, it we observe that the conjectures described above hold in all Boolean Glass networks with equilibrium nodes and with cyclic attractors.

While the cubes with cyclic attractors ensure the existence of the Glass PLDE with stable periodic orbits along the attractors, periodic phase flow may also exist in the networks without cyclic attractors. In such networks, the analysis of the conjecture about periodic orbits requires an additional step, as the criteria for periodic orbits [12] have to be checked. For example, the wiring schemes $1 \longrightarrow 2 \longrightarrow 3 \longrightarrow 1$ and $1 \longrightarrow 2 \longrightarrow 3 \longrightarrow 1$ constitute positive circuits and are induced by the transition diagrams with simple cycles of length six (see Appendix). The existence of a Glass network with a stable periodic orbit and one of these transition diagrams would be a counterexample for the conjecture in the case of PLDE.

\section{CONSTRUCTION OF Glass Networks WITH PERIODIC ORBITS}

The construction of the Glass PLDE with periodic orbits reported in the literature relies on random specification of the focal coordinates [15] and subsequent numerical calculations that check the criteria [12]. The conclusions based on the calculations are limited by the rounding errors introduced during the numerical procedure. In this final section, we therefore suggest an algebraic method for the construction of Glass PLDE with periodic orbits that avoids the complications connected with numerical methods and the ambiguity of the random choice of the focal points.

In the Glass model, the transition diagrams define only the signs of the focal points of the Glass PLDE but do not specify the phase flow unambiguously. A perturbation of the absolute values of the focal coordinates may lead to a bifurcation between periodic orbits and a flow converging to the origin [1]. If the signs of the focal coordinates are fixed and only absolute values of the coordinates are perturbed, the Glass PLDE will possess the same transition diagram. We exploit this observation to perform synthesis of Glass networks with periodic orbits.

We assume that the given transition diagram has a cycle that is meant to define a periodic phase flow. Our construction is based on the criteria for periodic flow in the Glass PLDE [12]. Let $L$ denote the length of the cycle, i.e., the number of the nodes of this cycle. Let $\boldsymbol{f}^{+}=\left(\boldsymbol{f}^{+(1)}, \ldots, \boldsymbol{f}^{+(L)}\right)$ be a sequence of unknown absolute values of the focal coordinates $\boldsymbol{f}^{(k)}$ and let $s=\left(\operatorname{sign}\left(f_{i}^{(1)}\right), \ldots, \operatorname{sign}\left(f_{i}^{(L)}\right)\right)$ be the sequence of the signs of the coordinates defined by the given diagram. Based on the criteria given in [12], we introduce the following set of equalities and inequalities that are satisfied iff a periodic flow exists:

1) Let $\boldsymbol{y}(k)$ denote the coordinate vector of a phase trajectory when it crosses the face of the orthant that corresponds to the hypercube edge connecting nodes $k$ and $k+1$. The coordinates are calculated iteratively [12]:

$$
y_{i}^{(k+1)}=\frac{f_{i}^{(k)} y_{j}^{(k)}-f_{j}^{(k)}}{y_{j}^{(k)}-f_{j}^{(k)}},
$$

where $j$ indicates the variable that is switching on the exit boundary for this orthant (i.e., $y_{j}^{(k+1)}=0$ ).

2) The faces of the orthant crossed by the flow along the cycle are specified by

$$
y_{j}^{(k)}=0 ; \quad O^{(k)} \tilde{\boldsymbol{y}}^{(k)}>0,
$$

where $\tilde{\boldsymbol{y}}^{(k)}$ is the vector $\boldsymbol{y}^{(k)}$ without coordinate $y_{j}^{(k)}$, and $O^{(k)}$ is the $(n-1) \times(n-1)$ diagonal matrix defining the signs of the coordinates on the face of the orthant with $O_{i i}^{(k)}= \pm 1$. The system of inequalities written for all $k$ is equivalent to the returning cone condition [12] that insures flow along the nodes of the cycle.

3) Following the notation of [12], let $A$ denote the matrix that is associated with the Poincare map on the starting face of the orthant that is specified by the first node of the hypercube cycle. If the Glass PLDE has a periodic orbit along the cycle, the staring point $\boldsymbol{y}^{(1)}$ is a fixed point of the Poincare map and is computed as follows:

$$
\boldsymbol{y}^{(1)}=\frac{(\lambda-1) v}{\langle\phi, v\rangle},
$$

where $\lambda$ and $v$ is the eigenvalue and the eigenvector of $A$, respectively, and the elements of the matrix $A$ and the vector $\phi$ can be analytically calculated as functions of $\boldsymbol{f}^{+}$for any given sign sequence $s$ [12].

4) The eigenvalue has to be real and greater than unity:

$$
\lambda>1 \text {. }
$$

The additional condition that $\lambda$ is the dominant eigenvalue guarantees asymptotic stability of the orbit.

The equalities and inequalities (2-5) can be seen as a system of constraints on the set of $n \cdot L$ unknown parameters $f_{i}^{+(k)}$, and of $n-1$ coordinates $\tilde{y}_{i}^{(1)}$ of the fixed point. For example, the inequality (5) does not require the computation of the eigenvalue but serves as a constraint on the coefficients of the characteristic polynomial $|A-\lambda E|=0$. If the system (2-5) is consistent, there is a Glass PLDE that possesses a periodic orbit, and any valuation of $\boldsymbol{f}^{+}, \tilde{\boldsymbol{y}}^{(1)}$ satisfying the system defines both the Glass PLDE and the initial conditions of the periodic orbit.

We have conducted an experimental evaluation of the method suggested above, utilizing the results of our classification of the three-dimensional Glass PLDE with 1-period orbits. The search for an instance satisfying the system (2-5) was implemented in Mathematica utilizing the subroutines for cylindrical decomposition. The identification of the PLDE with the periodic orbits was carried out for three transition diagrams with the cycles of length six shown in Figures 11, 12, and 13 , respectively. Due to memory restrictions of the machine used, the search was conducted only with two unknown focal coordinates. The absolute values of all other focal coordinates 


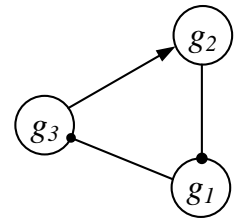

(a) Wiring diagram

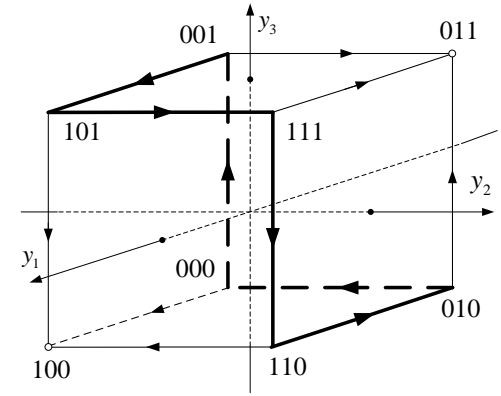

(b) Boolean Glass network
Fig. 11. A wiring scheme with a positive circuit and the unique Boolean Glass network complying to it. The network has a 6-coil (bold arrows) and 2 equilibria (hollow nodes)

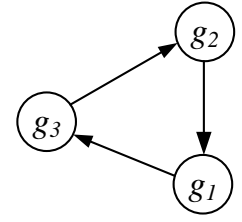

(a) Wiring diagram

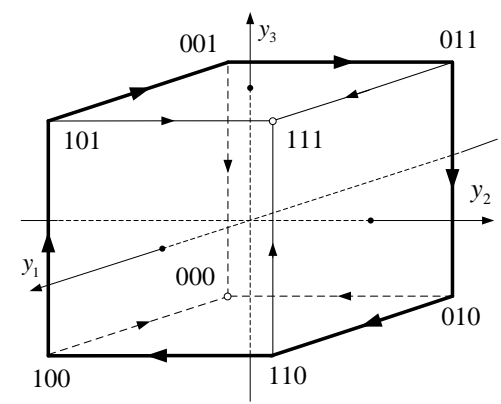

(b) Boolean Glass network
Fig. 12. A wiring scheme with a positive circuit and the unique Boolean Glass network complying to it.

were fixed and of equal unity. Thus, the synthesis of the PLDE was limited to the simultaneous perturbation of two focal coordinates of a Boolean Glass network that was defined by the given transition diagram. During the search, we checked all 153 possible combinations of two perturbed coordinates, out of 18 focal coordinates of the nodes of the cycles.

In the first two cases, the search resulted in 12 Glass PLDE with periodic orbits, and in the third case (see Figure 13), 11 such networks were identified. Tables VII, VIII and IX in the Appendix list the focal points of the PLDE and the initial conditions of the periodic orbits we found. Our results indicate that the perturbation of two coordinates in other than the listed cases cannot produce periodic orbits. In the third

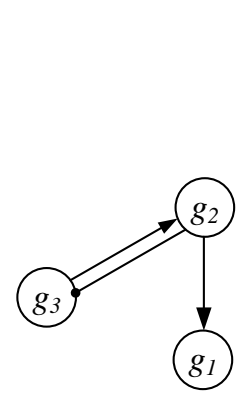

(a) Wiring diagram

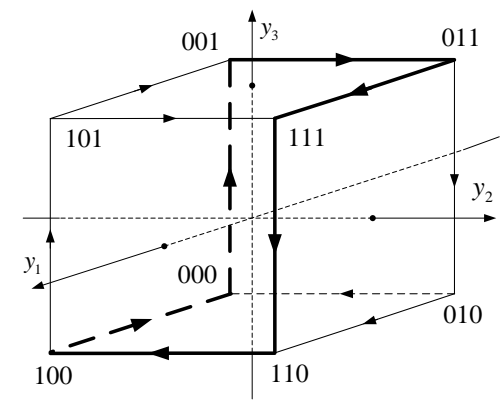

(b) Boolean Glass network
Fig. 13. A wiring scheme and the unique Boolean Glass network complying to it. case, four of 11 periodic orbits appeared to be asymptotically stable. In the first two cases, all orbits found are asymptotically unstable, and the results show that no stable orbits can be constructed by any two-coordinate perturbation of the given Boolean PLDE. Note that existence of a stable periodic orbit in the first or in the second case would have contradicted the conjecture about stable periodic solutions, as both the first and the second wiring schemes are the positive circuits.

\section{CONCLUSION AND Future WORK}

In this paper, we have introduced two new classes of complex attracting sets for the Glass PLDE. We have also suggested a classification of the dynamics in Glass networks with respect to their wiring schemes, which are a standard model for the interaction among genes. By means of a reduction of the search for the attractors to a SAT-based search for paths through binary hypercubes, we have obtained the full classification of the cyclic attractors up to dimension six for wiring schemes with three genes and three interactions.

The paper suggests a method for constructing the Glass transition diagrams for a given wiring scheme. We have applied this technique to a gene network regulating the cell division of fission yeast. An algebraic method of the construction of the Glass PLDE with periodic orbits along given cycles of the transition diagrams was proposed and tested.

It was found that the results obtained for the Glass PLDE comply with the conjectures [20] formulated for ODE with continuous coefficients. This consistency may be rooted in the fact that the Glass PLDE can be seen as a limit of the ODE with the continuous sigmoidal coefficients approaching step functions.

\section{Future Work}

We will investigate further ways to construct counterexamples for the conjectures for the ODE case [20]. In the Glass model, multiple equilibrium nodes as well as cyclic attractors in the hypercubes constitute sufficient conditions of the existence of multistationarity and stable periodic orbits, respectively. Therefore, if the conjectures hold, the corresponding statements about the transition diagrams of the Glass model must hold as well. We suggest to formulate these statements in the form of conjectures about oriented hypercubes with labeled nodes and their wiring schemes, following the terminology of the conjectures [20]:

1) The presence of a positive circuit (at least for one node of oriented hypercube) is a necessary condition for multiple equilibrium nodes in the hypercube.

2) If the oriented hypercube possesses multiple equilibrium nodes, its wiring schemes contain a variable nucleus or else the presence of two nuclei of opposite signs.

3) The presence of a negative circuit (at least for one node of oriented hypercube) is a necessary condition for the existence of a cyclic attractor.

4) Complex cycles in hypercubes of dimension higher than three require both a positive and a negative circuit.

The last conjecture reflects the fact that the Glass networks cannot have chaotic behavior in dimensions lower than four 
and that pseudo-chaotic dynamics is usually observed in those systems with complex cycles [16].

We have preliminary results for the application of the SAT-based algorithm presented in this paper to search for counterexamples for those conjectures. For example, conjecture 1 was found to hold in dimension three and four. For a more comprehensive study of the conjectures related to stable periodic orbits and chaotic dynamics, we plan to develop a decision procedure that combines a propositional SAT procedure (for generating the transition diagrams with complex cycles) together with algorithms for cylindrical decomposition (to identify the Glass PLDE with periodic orbits along the cycle).

\section{ACKNOWLEDGEMENTS}

We would like to thank Jasmin Fisher for stimulating discussions about gene regulatory networks, in particular in connection with the application to $C$. elegans.

\section{REFERENCES}

[1] I. Zinovik, D. Kroening, and Y. Chebiryak, "An algebraic algorithm for the identification of Glass networks with periodic orbits along cyclic attractors," in Algebraic Biology. Second International Conference, $A B$ 2007, ser. LNCS, vol. 4545, 2007, pp. 140-154.

[2] Y. Chebiryak, T. Wahl, D. Kroening, and L. Haller, "Finding lean induced cycles in binary hypercubes," in Theory and Applications of Satisfiability Testing. Springer, 2009, to appear.

[3] L. Glass and S. Kauffman, "The logical analysis of continuous nonlinear biochemical control networks," J. Theor. Biol., vol. 39, pp. $103-$ 129, 1973.

[4] L. Glass, "Combinatorial aspects of dynamics in biological systems," in Stat. Mech Stat. Methods in Theory and Application, U. Landman, Ed. Plenum Press, 1977, pp. 585-611.

[5] R. Edwards, "Symbolic dynamics and computation in model gene networks," Chaos, vol. 11, no. 1, pp. 160-169, 2001.

[6] R. Ghosh, A. Tiwari, and C. Tomlin, "Automated symbolic reachability analysis; with application to delta-notch signalic automata." in Hybrid Systems: Computation and Control, ser. LNCS, vol. 2623, 2003, pp. 233-248.

[7] J. Mason, P. Linsay, J. Collins, and L. Glass, "Evolving complex dynamics in electronic models of genetic networks," Chaos, vol. 14, no. 3, pp. 707-715, 2004.

[8] G. Batt, D. Ropers, H. de Jong, J. Geiselmann, R. Mateescu, M. Page, and D. Schneider, "Analysis and verification of qualitative models of genetic regulatory networks: A model-checking approach," in $19^{\text {th }}$ Int Joint Conference on Artificial Intelligence, 2005, pp. 370-375.

[9] T. Gedeon, "Global dynamics of neural nets with infinite gain," Physica D: Nonlinear Phenomena, vol. 146, pp. 200-212, 2000.

[10] - "Attractors in continuous time switching networks," Communications on Pure and Applied Analysis, vol. 2, no. 2, pp. 187-209, 2003.

[11] H. de Jong, "Modeling and simulation of genetic regulatory systems: a literature review," J. Comp. Biol., vol. 9, no. 1, pp. 67-103, 2002.

[12] R. Edwards, "Analysis of continuous-time switching networks," Physica $D$, vol. 146, pp. 165-199, 2000.

[13] L. Glass and J. Pasternack, "Stable oscillations in mathematical models of biological control systems," J. Math. Biol., vol. 6, pp. 207-223, 1978.

[14] T. Mestl, E. Plahte, and S. Omholt, "Periodic solutions in systems of piecewise-linear differential equations," Dynam. Stabil. Syst., vol. 10, no. 2, pp. 179-193, 1995.

[15] R. Edwards and L. Glass, "Combinatorial explosion in model gene networks," Chaos, vol. 10, no. 3, pp. 691-704, 2000.

[16] T. Mestl, C. Lemay, and L. Glass, "Chaos in high-dimensional neural and gene networks," Physica D, vol. 98, pp. 33-52, 1996.

[17] R. Thomas and M. Kaufman, "Multistationarity, the basis of cell differentiation and memory," Chaos, vol. 11, no. 1, pp. 170-195, 2001.

[18] S. Kauffman, "A proposal for using the ensemble approach to understand genetic regulatory networks," Theor. Biol., vol. 230, pp. 581-590, 2004.

[19] H. de Jong and M. Page, "Search for steady states of piecewise-linear differential equation models of genetic regulatory networks," IEEE/ACM Trans on Comput Biol and Bioinformatics, vol. 5, pp. 508-522, 2008.
[20] M. Kaufman, C. Soule, and R. Thomas, "A new necessary condition on interaction graphs for multistationarity," J Theoretical Biology, vol. 248 , no. 4, pp. 675-685, 2007.

[21] N. Eén and N. Sörensson, "An extensible SAT-solver," in Theory and Applications of Satisfiability Testing, ser. LNCS. Springer, 2004, vol. 2919, pp. 502-518.

[22] B. Dutertre and L. de Moura, "A fast linear-arithmetic solver for DPLL(T)," in CAV 2006, LNCS 4144. Springer, 2006, pp. 81-94.

[23] N. Kam, D. Harel, H. Kugler, R. Marelly, A. Pnueli, E. Hubbard, and M. Stern, "Formal modeling of C. elegans development: A scenariobased approach," in First International Workshop on Computational Methods in Systems Biology, ser. LNCS, vol. 2602, 2003, pp. 40-20.

[24] L. Glass and J. Pasternack, "Prediction of limit cycles in mathematical models of biological oscillations," Bull. Math. Biol., vol. 40, pp. 27-44. 1978.

[25] I. Zinovik, D. Kroening, and Y. Chebiryak, "Computing binary combinatorial Gray codes via exhaustive search with SAT-solvers," IEEE Trans Inf Theory, vol. 54, pp. 1819-1823, 2008.

[26] T. Perkinsa, M. Halletta, and L. Glass, "Inferring models of gene expression dynamics," J Theoret Biol, vol. 230, pp. 289-299, 2004.

[27] E. Farcot and J.-L. Gouze, "Periodic solutions of piecewise affine gene network models with non uniform decay rates using monotonicity properties," Acta Biotheoretica, vol. -, p. accepted, 2007.

[28] P. Sternberg, "Vulval development," WormBook, vol. 1, no. 1, June, 2005. [Online]. Available: http://www.wormbook.org

[29] O. Cinquin and J. Demongeot, "Positive and negative feedback: striking a balance between necessary antagonists," Journal of Theoretical Biology, vol. 216, no. 2, pp. 229-242, 2002.

[30] B. Novak, Z. Pataki, A. Ciliberto, and J. Tyson, "Mathematical model of the cell division cycle of fission yeast," Chaos: An Interdisciplinary Journal of Nonlinear Science, vol. 11, p. 277, 2001.

[31] C. Papadimitriou, Computational complexity. Addison-Wesley Reading, Mass, 1994.

[32] J. Szwarcfiter and P. Lauer, "A search strategy for the elementary cycles of a directed graph," BIT Numerical Mathematics, vol. 16, no. 2, pp. 192-204, 1976.

[33] D. Johnson, "Finding all the elementary circuits of a directed graph," SIAM Journal on Computing, vol. 4, p. 77, 1975.

[34] R. Tarjan, "Depth-first search and linear graph algorithms," SIAM Journal on Computing, vol. 1, no. 2, pp. 146-160, 1972. 


\section{APPENDIX}

\section{ClassificATION OF WIRING SCHEMES WITH 3 EDGES}

TABLE V

WIRING SCHEMES WITH 3 EDGES ALONG WITH TYPES OF GLASS NETWORKS COMPLYING TO THEM

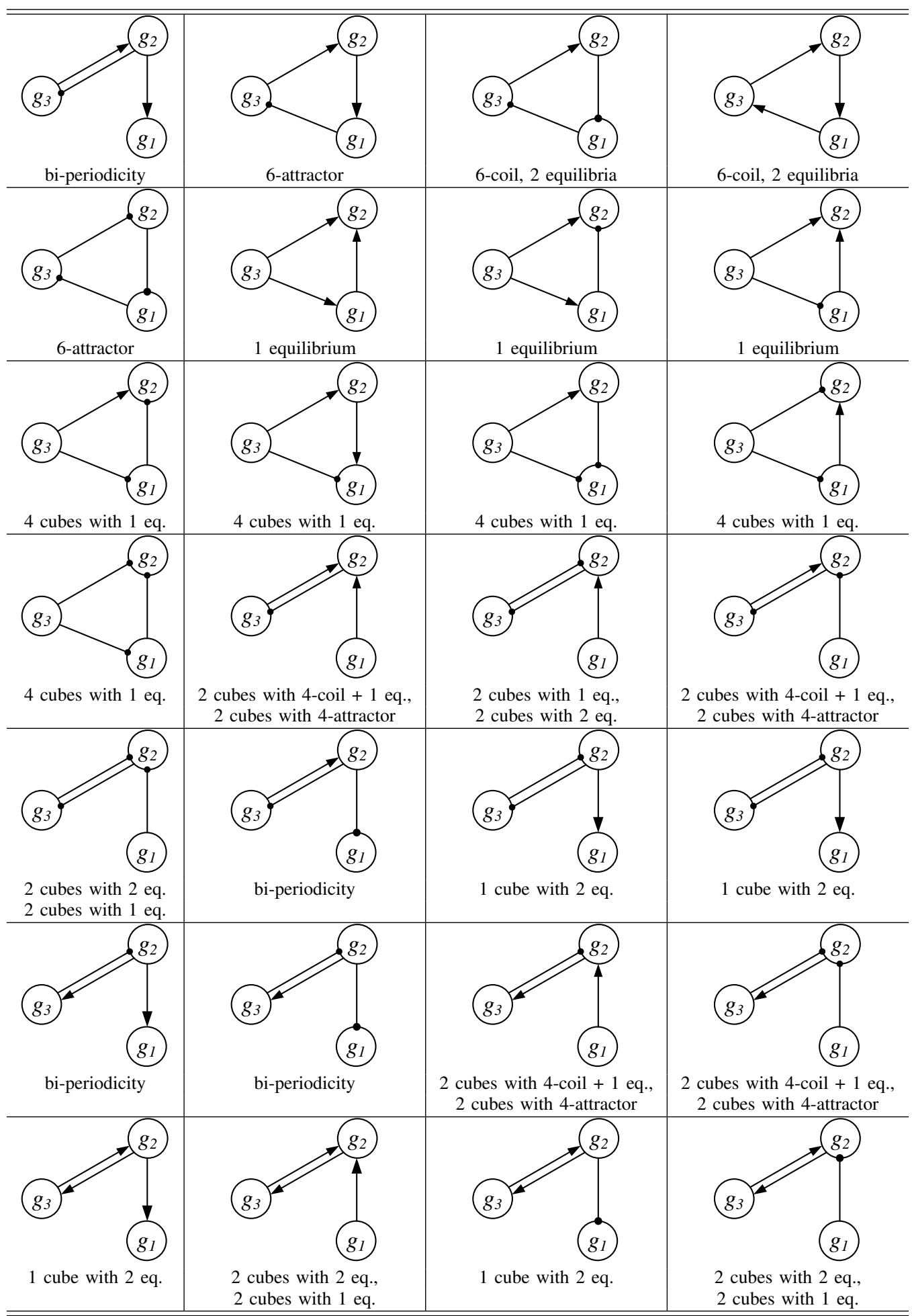


GLASS MODELS WITH PERIODIC ORBITS CORRESPONDING FIG. 9

Table VI presents results of the run of our algorithm on cubes complying to the scheme in Fig. 9 for all lengths of cycles from 4 to 32 . We have obtained all such cubes within 16 hours.

TABLE VI

ALl POSSIBLE CONFIGURATIONS

\begin{tabular}{|c|c|c|c|c|c|c|c|}
\hline$\overline{\mathrm{L}}$ & \#equilibria & \#complex cycles & \#cubes & $\mathrm{L}$ & \#equilibria & \#complex cycles & \#cubes \\
\hline \multirow[t]{9}{*}{4} & 0 & $\begin{array}{l}2 \\
\end{array}$ & 16 & \multirow[t]{6}{*}{14} & 0 & $\begin{array}{ll}2 \\
2\end{array}$ & 16 \\
\hline & 0 & 3 & 7 & & 0 & 3 & 7 \\
\hline & 0 & 4 & 6 & & 0 & 4 & 6 \\
\hline & 1 & 1 & 25 & & 1 & 3 & 1 \\
\hline & 1 & 2 & 15 & & 1 & 4 & 2 \\
\hline & 1 & 3 & 4 & & 1 & 5 & 1 \\
\hline & 1 & 4 & 3 & \multirow[t]{6}{*}{16} & 0 & 2 & 9 \\
\hline & 1 & 5 & 1 & & 0 & 3 & 7 \\
\hline & 2 & 2 & 4 & & 0 & 4 & 6 \\
\hline \multirow[t]{9}{*}{6} & 0 & 2 & 16 & & 1 & 3 & 1 \\
\hline & 0 & 3 & 7 & & 1 & 4 & 2 \\
\hline & 0 & 4 & 6 & & 1 & 5 & 1 \\
\hline & 1 & 1 & 25 & \multirow[t]{6}{*}{18} & 0 & 2 & 7 \\
\hline & 1 & 2 & 15 & & 0 & 3 & 6 \\
\hline & 1 & 3 & 4 & & 0 & 4 & 6 \\
\hline & 1 & 4 & 3 & & 1 & 3 & 1 \\
\hline & 1 & 5 & 1 & & 1 & 4 & 2 \\
\hline & 2 & 2 & 4 & & 1 & 5 & 1 \\
\hline \multirow[t]{8}{*}{8} & 0 & 2 & 16 & \multirow[t]{6}{*}{20} & 0 & 2 & 7 \\
\hline & 0 & 3 & 7 & & 0 & 3 & 7 \\
\hline & 0 & 4 & 6 & & 0 & 4 & 6 \\
\hline & 1 & 1 & 25 & & 1 & 3 & 1 \\
\hline & 1 & 2 & 7 & & 1 & 4 & 2 \\
\hline & 1 & 3 & 4 & & 1 & 5 & 1 \\
\hline & 1 & 4 & 3 & \multirow[t]{6}{*}{22} & 0 & 2 & 3 \\
\hline & 1 & 5 & 1 & & 0 & 3 & 3 \\
\hline \multirow[t]{4}{*}{10} & 0 & 2 & 16 & & 0 & 4 & 6 \\
\hline & 0 & 3 & 7 & & 1 & 3 & 1 \\
\hline & 0 & 4 & 6 & & 1 & 4 & 2 \\
\hline & 1 & 1 & 4 & & 1 & 5 & 1 \\
\hline \multirow[t]{7}{*}{12} & 0 & 2 & 7 & \multirow[t]{3}{*}{24} & 0 & 2 & 3 \\
\hline & 0 & 3 & 5 & & 0 & 3 & 3 \\
\hline & 0 & 4 & 6 & & 0 & 4 & 6 \\
\hline & 1 & 1 & 4 & 26 & 0 & 2 & 1 \\
\hline & 1 & 3 & 1 & & 0 & 4 & 3 \\
\hline & 1 & 4 & 2 & & & & \\
\hline & 1 & 5 & 1 & & & & \\
\hline
\end{tabular}


TABLE VII

PERIODIC ORBITS WITH WS: $1 \longrightarrow 2 \longrightarrow 3-\bullet$; FOCAL POINTS ALONG CYCLE IN TRANSITION DIAGRAM OF INITIAL BOOLEAN GLASS PLDE $f_{i}^{(k)}=((1$, $-1,1),(1,1,1),(1,1,-1),(-1,1,-1),(-1,-1,-1),(-1,-1,1))$, SHOWN FIXED POINT IS LOCATED ON ORTHANT FACE $y_{1}^{*}<0 ; y_{2}^{*}=0 ; y_{3}^{*}<0$

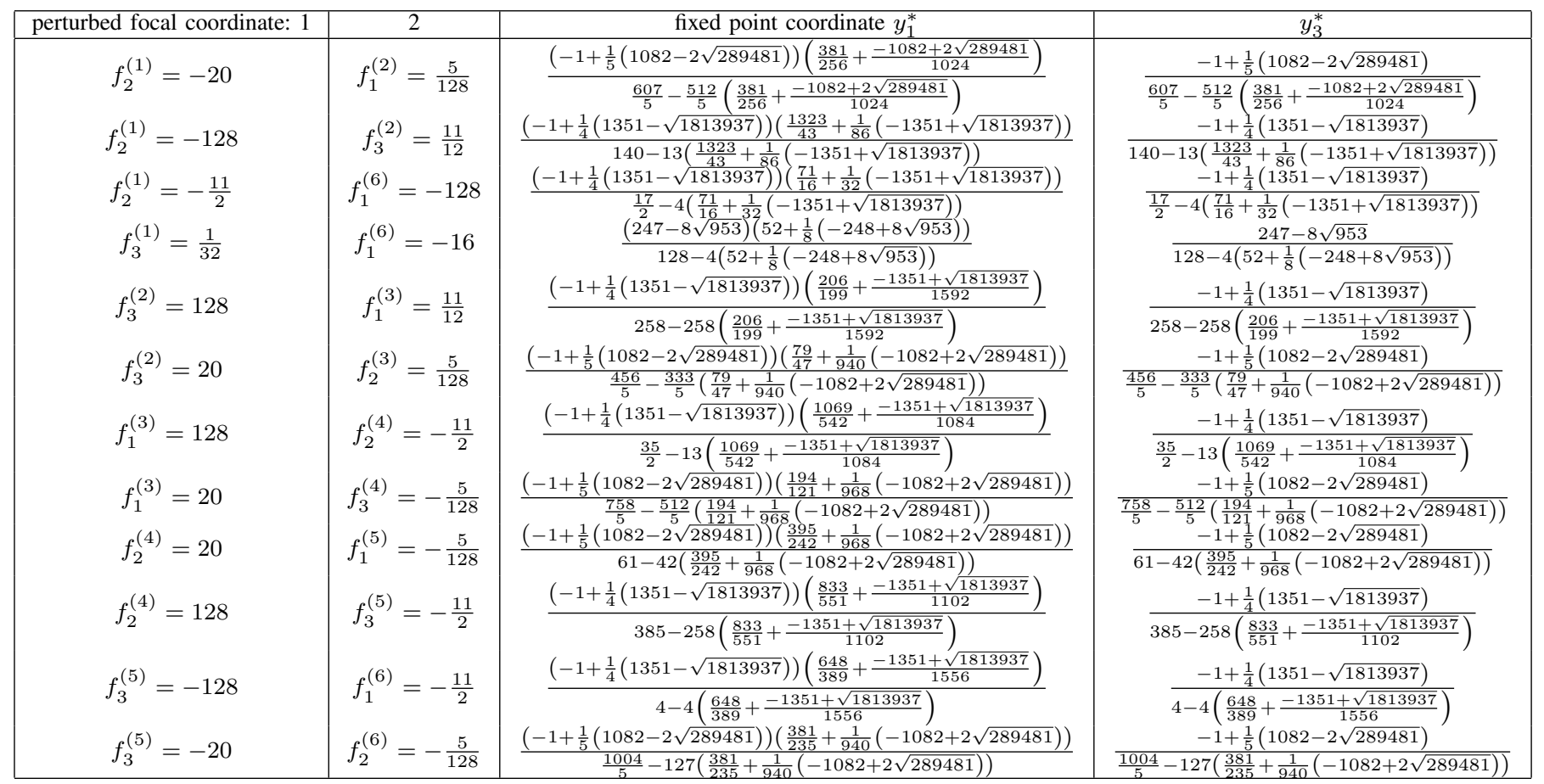

TABLE VIII
PERIODIC ORBITS WITH WS: $1 \longrightarrow 2 \longrightarrow 3 \longrightarrow 1$; FOCAL POINTS ALONG CYCLE IN TRANSITION DIAGRAM OF INITIAL BOOLEAN GLASS PLDE $f_{i}^{(k)}=((-1$, $1,1),(-1,1,-1),(1,1,-1),(1,-1,-1),(1,-1,1),(-1,-1,1))$, SHOWN FIXED POINT IS LOCATED ON ORTHANT FACE $y_{1}^{*}>0 ; y_{2}^{*}<0 ; y_{3}^{*}=0$

\begin{tabular}{|c|c|c|c|}
\hline perturbed focal coordinate: 1 & 2 & fixed point coordinate $y_{1}^{*}$ & $y_{2}^{*}$ \\
\hline$f_{1}^{(1)}=-\frac{1}{32}$ & $f_{2}^{(6)}=-16$ & $\frac{(247-8 \sqrt{953})\left(-\frac{5}{256}+\frac{248-8 \sqrt{953}}{4096}\right)}{548-8 \sqrt{953})}$ & $\frac{247-8 \sqrt{953}}{5 \sqrt{248-8 \sqrt{953}})}$ \\
\hline & 2 & $-4-128\left(-\frac{5}{256}+\frac{248-8 \sqrt{953}}{4096}\right)$ & $-4-128\left(-\frac{5}{256}+\frac{248-8 \sqrt{953}}{4096}\right)$ \\
\hline$f^{(1)}=128$ & $f^{(2)}=-\underline{11}$ & $\left(-\frac{7}{199}+\frac{1351-\sqrt{1813937}}{1592}\right)\left(-1+\frac{1}{4}(1351-\sqrt{1813937})\right)$ & $-1+\frac{1}{4}(1351-\sqrt{1813937})$ \\
\hline$J_{3}=128$ & $J_{1}=-\frac{\overline{2}}{2}$ & $-13-140\left(-\frac{7}{199}+\frac{1351-\sqrt{1813937}}{1592}\right)$ & $\overline{-13-140\left(-\frac{7}{199}+\frac{1351-\sqrt{1813937}}{1592}\right)}$ \\
\hline$f_{3}^{(1)}=20$ & $f_{2}^{(2)}=\frac{5}{128}$ & $\frac{\left(-\frac{32}{47}+\frac{1}{940}(1082-2 \sqrt{289481})\right)\left(-1+\frac{1}{5}(1082-2 \sqrt{289481})\right)}{-\frac{512}{5}-\frac{607}{5}\left(-\frac{32}{47}+\frac{1}{940}(1082-2 \sqrt{289481})\right)}$ & $\frac{-1+\frac{1}{5}(1082-2 \sqrt{289481})}{-\frac{512}{5}-\frac{607}{5}\left(-\frac{32}{47}+\frac{1}{940}(1082-2 \sqrt{289481})\right)}$ \\
\hline$f_{3}^{(1)}=\frac{11}{2}$ & $f_{2}^{(6)}=-128$ & $\frac{\left(-\frac{10}{43}+\frac{1351-\sqrt{1813937}}{11008}\right)\left(-1+\frac{1}{4}(1351-\sqrt{1813937})\right)}{-4-\frac{17}{2}\left(-\frac{10}{43}+\frac{1351-\sqrt{1813937}}{11008}\right)}$ & $\frac{-1+\frac{1}{4}(1351-\sqrt{1813937})}{-4-\frac{17}{2}\left(-\frac{10}{43}+\frac{1351-\sqrt{1813937}}{11008}\right)}$ \\
\hline$f^{(2)}=-128$ & $f^{(3)}=\underline{11}$ & $\left(-\frac{527}{542}+\frac{1351-\sqrt{1813937}}{1084}\right)\left(-1+\frac{1}{4}(1351-\sqrt{1813937})\right)$ & $-1+\frac{1}{4}(1351-\sqrt{1813937})$ \\
\hline$J_{1}--120$ & $J_{2}-\overline{2}$ & $-258-258\left(-\frac{527}{542}+\frac{1351-\sqrt{1813937}}{1084}\right)$ & $\overline{-258-258\left(-\frac{527}{542}+\frac{1351-\sqrt{1813937}}{1084}\right)}$ \\
\hline$f_{1}^{(2)}=-20$ & $f_{3}^{(3)}=-\frac{5}{128}$ & $\left(-\frac{73}{121}+\frac{1}{968}(1082-2 \sqrt{289481})\right)\left(-1+\frac{1}{5}(1082-2 \sqrt{289481})\right)$ & $-1+\frac{1}{5}(1082-2 \sqrt{289481})$ \\
\hline & & $\begin{array}{c}-\frac{333}{5}-\frac{456}{5}\left(-\frac{73}{121}+\frac{1}{968}(1082-2 \sqrt{289481})\right) \\
\left(-\frac{153}{242}+\frac{1}{068}(1082-2 \sqrt{289481})\right)\left(-1+\frac{1}{5}(1082-2 \sqrt{289481})\right)\end{array}$ & $\begin{array}{c}-\frac{333}{5}-\frac{456}{5}\left(-\frac{73}{121}+\frac{1}{968}(1082-2 \sqrt{289481})\right) \\
-1+\frac{1}{5}(1082-2 \sqrt{289481})\end{array}$ \\
\hline$=20$ & $f_{1}^{(4)}$ & $\frac{-512}{5}-\frac{758}{5}\left(-\frac{153}{242}+\frac{1}{968}(1082-2 \sqrt{289481})\right)$ & $-\frac{512}{5}-\frac{758}{5}\left(-\frac{1533}{242}+\frac{1}{968}(1082-2 \sqrt{289481})\right)$ \\
\hline$f_{2}^{(3)}=128$ & $f_{3}^{(4)}=-\frac{11}{2}$ & $\underline{\left(-\frac{282}{551}+\frac{1351-\sqrt{1813937}}{1102}\right)\left(-1+\frac{1}{4}(1351-\sqrt{1813937})\right)}$ & $-1+\frac{1}{4}(1351-\sqrt{1813937})$ \\
\hline & $\begin{array}{ll}3 & 2\end{array}$ & $-13-\frac{35}{2}\left(-\frac{282}{551}+\frac{1351-\sqrt{1813937}}{1102}\right)$ & $-13-\frac{35}{2}\left(-\frac{282}{551}+\frac{1351-\sqrt{1813937}}{1102}\right)$ \\
\hline$f_{1}^{(4)}=128$ & $f_{1}^{(6)}=-\frac{11}{2}$ & $\frac{\left(-\frac{66}{131}+\frac{2}{131}(229-\sqrt{52433})\right)\left(-1+\frac{1}{32}(229-\sqrt{52433})\right)}{-\frac{1}{20}+\frac{125}{25}\left(-\frac{66}{12}+\frac{2}{129}(229-\sqrt{52433})\right)}$ & $\frac{-1+\frac{1}{32}(229-\sqrt{52433})}{\left.-\frac{1}{3}+\frac{125}{66}+\frac{2}{2}(229-\sqrt{52433})\right)}$ \\
\hline$f^{(4)}=20$ & $f^{(6)}=-\frac{5}{5}$ & $\left(-1+\frac{1}{640}(1439-\sqrt{2069921})\right)\left(-\frac{12}{23}+\frac{2}{115}(1439-\sqrt{2069921})\right)$ & $\begin{array}{c}32+64+\frac{1}{640}(1439-\sqrt{2069921}) \\
-1+140\end{array}$ \\
\hline$J_{1}-20$ & & $\begin{array}{c}-\frac{1}{5}+\frac{17}{10}\left(-\frac{12}{23}+\frac{2}{115}(1439-\sqrt{2069921})\right) \\
\left(-\frac{5}{8}+\frac{1}{176}(1351-\sqrt{1813937})\right)\left(-1+\frac{1}{4}(1351-\sqrt{1813937})\right)\end{array}$ & $\begin{array}{c}-\frac{1}{5}+\frac{17}{10}\left(-\frac{12}{23}+\frac{2}{115}(1439-\sqrt{2069921})\right) \\
-1+\frac{1}{4}(1351-\sqrt{1813937})\end{array}$ \\
\hline & & $-4-4\left(-\frac{5}{8}+\frac{1}{176}(1351-\sqrt{1813937})\right)$ & $\overline{-4-4\left(-\frac{5}{8}+\frac{1}{176}(1351-\sqrt{1813937})\right)}$ \\
\hline$f_{1}^{(5)}=20$ & $f_{2}^{(6)}=\frac{5}{120}$ & $\underline{\left(-\frac{5}{8}+\frac{1082-2 \sqrt{289481}}{1024}\right)\left(-1+\frac{1}{5}(1082-2 \sqrt{289481})\right)}$ & $-1+\frac{1}{5}(1082-2 \sqrt{289481})$ \\
\hline & & $-127-\frac{1004}{5}\left(-\frac{5}{8}+\frac{1082-2 \sqrt{289481}}{1024}\right)$ & $-127-\frac{1004}{5}\left(-\frac{5}{8}+\frac{1082-2 \sqrt{289481}}{1024}\right)$ \\
\hline
\end{tabular}


TABLE IX

PERIODIC ORBITS WITH WS $: 2 \longrightarrow 1 \longrightarrow 2 \longrightarrow 3$; FOCAL POINTS ALONG CYCLE IN TRANSITION DIAGRAM OF INITIAL BOOLEAN GLASS PLDE $f_{i}^{(k)}=((-1,-1,1),(-1,1,1),(1,1,-1),(1,1,-1),(1,-1,-1),(-1,-1,1))$, SHOWN FIXED POINT IS LOCATED ON ORTHANT FACE $y_{1}^{*}=0 ; y_{2}^{*}<0 ; y_{3}^{*}<0$

\begin{tabular}{|c|c|c|c|}
\hline perturbed focal coordinate: 1 & 2 & fixed point coordinate $y_{2}^{*}$ & $y_{3}^{*}$ \\
\hline $\begin{aligned} f_{1}^{(2)} & =-\frac{25}{32} \\
f_{1}^{(2)} & =-\frac{1}{4} \\
f_{3}^{(2)} & =\frac{5}{4} \\
f_{3}^{(2)} & =\frac{13}{8} \\
f_{3}^{(2)} & =\frac{5}{4} \\
f_{1}^{(3)} & =\frac{13}{8} \\
f_{1}^{(3)} & =\frac{5}{4} \\
f_{3}^{(3)} & =-\frac{3}{32} \\
f_{3}^{(3)} & =-\frac{3}{4} \\
f_{3}^{(3)} & =-\frac{3}{4} \\
f_{3}^{(3)} & =-\frac{3}{32}\end{aligned}$ & $\begin{aligned} f_{3}^{(5)} & =-\frac{5}{4} \\
f_{3}^{(6)} & =-\frac{3}{32} \\
f_{1}^{(5)} & =\frac{25}{32} \\
f_{3}^{(5)} & =-\frac{3}{2} \\
f_{3}^{(6)} & =\frac{3}{4} \\
f_{3}^{(5)} & =-2 \\
f_{3}^{(6)} & =\frac{3}{4} \\
f_{1}^{(5)} & =\frac{1}{4} \\
f_{3}^{(5)} & =-\frac{5}{4} \\
f_{1}^{(6)} & =-\frac{5}{4} \\
f_{3}^{(6)} & =\frac{1}{8}\end{aligned}$ & $\begin{array}{c}-\frac{7}{135} \\
\frac{\left(-1+\frac{1}{256}(343-\sqrt{52113})\right)\left(-\frac{119}{107}+\frac{1}{214}(343-\sqrt{52113})\right)}{-\frac{19}{4}-\frac{19}{4}\left(-\frac{119}{107}+\frac{1}{214}(343-\sqrt{52113})\right)} \\
-\frac{29}{605} \\
-\frac{5}{26} \\
\frac{\left(-1+\frac{1}{16}(19-\sqrt{41})\right)\left(-7+\frac{1}{2}(19-\sqrt{41})\right)}{-5-5\left(-7+\frac{1}{2}(19-\sqrt{41})\right)} \\
-\frac{5}{41} \\
\frac{\left(-1+\frac{1}{20}(19-\sqrt{41})\right)\left(-7+\frac{1}{2}(19-\sqrt{41})\right)}{-\frac{22}{5}-\frac{21}{5}\left(-7+\frac{1}{2}(19-\sqrt{41})\right)} \\
\frac{1-\sqrt{52113}))\left(-\frac{166}{67}+\frac{1}{134}(343-\sqrt{52113})\right)}{-\frac{585}{64}-\frac{793}{128}\left(-\frac{166}{67}+\frac{1}{134}(343-\sqrt{52113})\right)} \\
\frac{(5-\sqrt{41})\left(-1+\frac{1}{16}(19-\sqrt{41})\right)}{-6-5(5-\sqrt{41})} \\
\frac{(5-\sqrt{41})\left(-1+\frac{1}{20}(19-\sqrt{41})\right)}{-\frac{29}{5}-\frac{47}{10}(5-\sqrt{41})} \\
\frac{\left(-\frac{302}{199}+\frac{1}{199}(331-3 \sqrt{10353})\right)\left(-1+\frac{1}{128}(331-3 \sqrt{10353})\right)}{-\frac{45}{4}-\frac{61}{8}\left(-\frac{302}{109}+\frac{1}{199}(331-3 \sqrt{10353})\right)}\end{array}$ & $\begin{array}{c}-\frac{1}{135} \text { Stable } \\
\frac{-1+\frac{1}{256}(343-\sqrt{52113})}{-\frac{19}{4}-\frac{19}{4}\left(-\frac{119}{107}+\frac{1}{214}(343-\sqrt{52113})\right)} \\
-\frac{3}{605} \text { Stable } \\
-\frac{3}{104} \text { Stable } \\
\frac{-1+\frac{1}{16}(19-\sqrt{41})}{-5-5\left(-7+\frac{1}{2}(19-\sqrt{41})\right)} \\
-\frac{4}{41} \text { Stable } \\
\frac{-1+\frac{1}{20}(19-\sqrt{41})}{-\frac{22}{5}-\frac{21}{5}\left(-7+\frac{1}{2}(19-\sqrt{41})\right)} \\
\frac{-1+\frac{1}{256}(343-\sqrt{52113})}{-\frac{585}{64}-\frac{793}{128}\left(-\frac{166}{67}+\frac{1}{134}(343-\sqrt{52113})\right)} \\
\frac{-1+\frac{1}{16}(19-\sqrt{41})}{-6-5(5-\sqrt{41})} \\
\frac{-1+\frac{1}{20}(19-\sqrt{41})}{-\frac{29}{5}-\frac{47}{10}(5-\sqrt{41})} \\
-1+\frac{1}{128}(331-3 \sqrt{10353}) \\
\frac{45}{-\frac{41}{4}-\frac{61}{8}\left(-\frac{32}{109}+\frac{1}{199}(331-3 \sqrt{10353})\right)}\end{array}$ \\
\hline
\end{tabular}

